\title{
Attention modulates set representation by statistical properties
}

\author{
Jan W. De Fockert and Alexander P. Marchant \\ Goldsmiths, University of London, London, England
}

\begin{abstract}
Recent reports have claimed that observers show accurate knowledge of the mean size of a group of similar objects, a finding that has been interpreted to suggest that sets of multiple objects are represented in terms of their statistical properties, such as mean size (Ariely, 2001; Chong \& Treisman, 2003, 2005a, 2005b). In the present study, we directed visual attention to a single set member and found that mean estimations were modulated according to the size of the attended item, regardless of whether size was the relevant search criterion (Experiment 1) or not (Experiment 2). These findings suggest that observers do not always accurately average together the entire set, and that instead the average is either biased by the features of the attended item, or based on a short-cut strategy of extracting the mean of a smaller subset.
\end{abstract}

In our everyday visual experience, objects rarely occur in isolation; instead, they are often part of a set of multiple objects of the same category, such as a number of oranges in a fruit bowl or leaves on a tree. Recent findings have suggested that the visual system may represent such sets of similar items by abstracting a schematic summary in terms of its descriptive statistics, such as the mean or distribution. When observers are briefly shown displays consisting of sets of circles with varying sizes and are asked whether a subsequently presented single test circle was part of the previous set, they are most likely to indicate that the test circle was part of the set when its size is close to the mean size of the set, and least likely to indicate that the test circle was part of the set when its size is outside the range of the set (Ariely, 2001). By contrast, observers are no more likely to correctly identify an actual member of the previous set as they do a nonmember whose size is close to the mean of the set. These findings suggest that, when one is perceiving sets of similar items, the visual system does not represent each item in the way that it does a single item, but instead may represent the set in terms of its statistical parameters - in this case, mean size. This process has been termed set representation, and the processing of a set of similar objects by extraction of overall statistical properties of the set, rather than individual item properties, provides an efficient way of processing complex scenes.

The extraction of the statistical properties of a set of items appears to be a robust process, and it applies to many stimulus dimensions, including orientation (e.g., Dakin \& Watt, 1997; Parkes, Lund, Angelucci, Solomon, \& Morgan, 2001), motion speed (e.g., Atchley \& Andersen, 1995; Watamaniuk \& Duchon, 1992), and motion direction (Williams \& Sekuler, 1984). Moreover, the accuracy of judgments of mean size is almost as good as the accuracy of size judgments for a single item (Chong \& Treisman, 2003). Mean judgment accuracy also remains good under difficult perceptual conditions, such as brief set exposure duration, or the insertion of a delay between two sets, the means of which need to be compared. Increasing the number and density of the elements in a set also does not lead to a deterioration in mean judgment (Ariely, 2001; Chong \& Treisman, 2005b).

However, when processing large sets of similar items, we often search for (and find) one particular member of that set, rather than distribute our attention equally across all members of the set. It remains unclear precisely how this process of attending to a single set member affects the representation of the statistical parameters of the set. In the present study, we therefore investigated how mean judgments are affected when attention is directed toward a particular member of the set, and to what extent the effect is a function of the features of the attended item. We instructed participants to estimate the mean size of a set of circles while they directed their attention to either a small or a large target set member and reported the location of the target, before asking them to make the mean size judgment. We measured the mean size judgments as a function of the size of the attended item in the preceding set.

Previous evidence suggests that manipulations of attention can affect the accuracy of mean estimations (Chong \& Treisman, 2005a). Mean estimations are slightly more accurate when attention is globally allocated to the entire set of objects than when it is locally allocated to a location without a member of the set (the center of the screen). In addition, when attention is directed to a single member of the set (regardless of its size), mean estimations are more accurate when the item pops out, and attention can be distributed globally across the set, than when attention has

J.W. de Fockert, j.de-fockert@gold.ac.uk 
to be shifted serially between items before being focused on one single item (Chong \& Treisman, 2005a). Whereas these findings clearly suggest that attention can modulate set representation, making mean judgments less accurate when attention is focused on a specific location, they do not address the question of whether and how the statistical representation of a set of items may be affected by the value of the member of the set that receives attention.

There are at least three possible outcomes of manipulating attention to a single set item in terms of the computation of the statistical parameters of a set. First, it may be that mean judgments are not affected by this type of attention manipulation. Previous findings that set averaging is surprisingly resistant to increases in processing demands (e.g., Ariely, 2001; Chong \& Treisman, 2005b) such as reducing the exposure duration of the set support this possibility. The attention manipulation should have little effect on mean judgments if whole-set averaging occurs automatically, and the mean size is based on all or most of the members in a set regardless of any processing differences between set members. If whole-set averaging is indeed the robust process it has been suggested to be (e.g., Ariely, 2001), this should not be systematically affected by the features of the attended item.

Second, it may be that the addition of a task requiring attention to be directed to a single set member compromises the ability to process all or most of the set items. On this view, there should be a nonspecific cost in mean judgment accuracy in comparison with when attention can be distributed across the entire set, regardless of the features of the attended item. Alternatively, there may be a nonspecific cost in mean judgment accuracy due to the dual task requirement of having to report both the target location and the estimated mean size on each trial.

Third, attention to a single set member may modulate mean judgments in a systematic way, suggesting that the features of an attended item have a disproportionate influence on the extracted statistical set parameters. In terms of whole-set averaging, a systematic modulation of the statistical representation following attention to a small or large set member would suggest that mean judgments are still based on the majority of set items, but that the extracted mean size is shifted toward the size of the attended item. This may be because the attended item has been given more weight in the computation of the average size, or because the requirement to attend to a small or large set item has meant that observers tend to search set items toward that end of the size distribution, at the expense of items at the other end of the distribution.

An alternative explanation of a systematic modulation of the statistical representation following attention to a small or large set member is that the requirement to attend to a single set member has led to a strategy that involves making a mean judgment on the basis of a subset of items. On this view, observers use a strategy that does not involve extracting the mean of the entire set, but instead select a small subset of the set items to base their mean estimation on. Recent behavioral and simulation findings (Myczek \& Simons, 2008) indeed suggest such a mechanism, by showing that the same mean judgment performance as predicted by whole-set averaging can sometimes be obtained when only a small subset (one or two set items) is selected and mean judgments are made by comparing the test item with the selected item (if a single item was selected) or the average of the two selected items. In a task that required observers to judge whether the size of a test circle was smaller or greater than the mean size of a previously presented set of eight circles, actual performance was approximated most closely by a simulation in which a subset of either one or two set items was randomly selected, and mean judgments were made by comparing the test item with the selected subset.

In the present study, we hypothesized that a mechanism of subset averaging would lead to a systematic variation of the mean size of the subset when either the smallest or the largest set member would be part of the selected subset. We ensured that the subset contained either the smallest or the largest set member by directing attention to one of these items on each trial. Importantly, the mean of the subset was likely to be different from that of the entire set (the only situation in which mean estimations would be the same whether based on subset averaging or based on whole-set averaging is when the other member in a subset of two is the item at the other extreme of the distribution, which would occur only in $12.5 \%$ of cases, whereas in the other $87.5 \%$ the subset mean would be shifted toward the size of the attended target). Therefore, if observers perform the mean estimation task by comparing the size of a test item with the mean of a selected subset, their estimation should necessarily vary depending on whether the subset includes the smallest or largest set item.

Thus, the attention manipulation in the present study elicits different predictions from, on the one hand, a strong version of the whole-set averaging account (e.g., Ariely, 2001), and, on the other hand, either a weaker version of whole-set averaging, in which averaging still involves most of the set items, but the extracted mean can be modulated when all set members are not processed equally, or the subset averaging account. However, the original aim of the present study was to investigate how the features of an attended set item would affect the extracted statistical properties of the whole set, and we only became aware of Myczek and Simons's (2008) alternative explanation in terms of a short-cut strategy of subset sampling after we had collected our data. The present study therefore was not designed to differentiate between the more flexible version of whole-set averaging that allows for the predicted attention effect, and the alternative explanation in terms of subset averaging.

\section{EXPERIMENT 1}

In Experiment 1, participants were shown sets of nine heterogeneously sized circles and were asked to estimate the mean size of the entire set. On each trial, attention was directed to a target circle that was either the smallest or the largest circle in the display. Following the set, two test circles were presented: One circle had the same size as the mean size of the previous set, and the other circle was either smaller or larger than the mean. Participants 
were asked which circle had the same size as the mean size of the previous set, and we argued that, if whole-set averaging was susceptible to the attention manipulation, then attention to the smallest (vs. largest) set item would lead to a smaller (vs. larger) extracted mean. Similarly, if participants used a strategy of subset averaging, then attention to the smallest (vs. largest) set item would bias the probability of that item to be included in the subset, and therefore lead to a smaller (vs. larger) subset mean. Participants should therefore show a tendency to select the smaller (vs. larger) test circle on trials on which attention had previously been directed toward a small (vs. large) set item.

\section{Method}

Participants. Twenty-three undergraduate students ( 2 males; age range, 19-45; mean age, 26) from Goldsmiths, University of London participated in exchange for course credit. All had normal or corrected-to-normal vision.

Materials and Procedure. All stimuli were presented in light gray on a black background on a 17 -in. monitor, connected to a PC running E-Prime (Schneider, Eschman, \& Zuccolotto, 2002) software. Participants were seated approximately $60 \mathrm{~cm}$ from the screen. Each experimental trial started with the cue word "Small" or "Large" presented for $500 \mathrm{msec}$, which instructed participants to report the location (left or right) of either the smallest or the largest set member in the following set display. Following the cue word, a set of nine filled circles, each with a different diameter ranging from $0.8^{\circ}$ to $2.9^{\circ}$ of visual angle (size difference between circles, $0.2625^{\circ}$; mean diameter, $1.9^{\circ}$ ) was presented for $1,000 \mathrm{msec}$, and participants were asked to report the location of the target circle by pressing "c" or " $m$ " with their left or right index finger when they thought the target appeared on the left or right side of the display, respectively. The set display was a centered $3 \times 3$ matrix; and within each square matrix cell, a set circle was presented in one of the four quadrants of the cell in order to give the array a less regular appearance. This jitter was random, with the constraint that if the target circle appeared in the middle column, it would be the only circle within that column with a jitter to one side, whereas the other two would be jittered to the opposite side (without this constraint, the target location task would be more difficult for targets that appeared in the middle column). The size of the entire set display was approximately $12^{\circ} \times 12^{\circ}$. Following a 1,000 -msec blank screen, two test circles were presented for $3,000 \mathrm{msec}$ or until response. The test circles were presented $3.5^{\circ}$ to the left and right of the center of the screen. The size of one of the test circles was always the same as the mean size of the previous set; the other circle was equally likely to be either $13.8 \%$ smaller or $13.8 \%$ larger than the mean of the previous set. Both test circles were members of the previous set. Participants pressed "c" (left) or "m" (right), depending on which of the test circles (left or right) they thought had the same size as the mean of the previous set. The next trial was presented after a $500-\mathrm{msec}$ blank screen. The test circle with the same size as the mean set size was equally likely to occur on the left as on the right of the display, and equally likely to be on the same (vs. the other) side as the target circle in the preceding set. In line with previous studies (e.g., Chong \& Treisman, 2003, 2005a), we used stimulus sets of three different absolute sizes (with the sizes described above, or multiplied in diameter by either 0.9 or 1.1) in order to discourage participants from basing their judgments on the absolute size of previously presented test circles.

In order to ensure adequate ability to estimate the mean size of the set displays, participants first completed three blocks of 36 practice trials, in which they saw the set of circles (without the attention manipulation) followed by the test displays and were instructed to indicate which test circle they thought had the same mean size as that of the previously seen set. During practice, participants received feedback in the form of a short high-pitched tone if they chose the incorrect test circle or did not respond within a 3,000-msec response window. Participants were given the experimental trials only if they reached $60 \%$ accuracy during the final practice block. There was no feedback during the experimental trials.

\section{Results and Discussion}

Data from 1 participant who failed to respond to the target location task were removed from the analysis. Mean accuracy on the localization task was .77. Only trials on which the target circle was correctly located were included in the analysis of mean estimation accuracy. Overall mean estimation accuracy (.60) differed significantly from chance [one-sample $t$ test with test value $=.50 ; t(21)=$ $5.42, p<.001]$. Mean estimation accuracies were entered into a 2 (test circle size: large vs. small) $\times 2$ (target circle size: large vs. small) repeated measures ANOVA, with participants as the random factor (see Figure 1, left graph). There was no main effect of test circle size $(F<1)$, suggesting that mean estimation accuracy was equal for large $(M=.595, S E=.035)$ and small $(M=$ $.605, S E=.034)$ test circles. There was a significant main effect of target circle size $\left[F(1,21)=6.55, M S_{\mathrm{e}}=.004\right.$, $p<.05]$. Mean estimations were more accurate following a large target circle $(M=.618, S E=.023)$ than following a small target circle $(M=.581, S E=.016)$. Importantly, there was a significant interaction between test circle size and target circle size $\left[F(1,21)=5.01, M S_{\mathrm{e}}=.072, p<\right.$ $.05]$. Mean estimations for large test circles were more accurate following a large target circle $(M=.677, S E=$ $.044)$ than following a small target circle $(M=.512, S E=$ $.046)[t(21)=2.85, p<.02]$. Numerically, mean estimations for small test circles were more accurate following a small target circle $(M=.650, S E=.039)$ than following a large target circle $(M=.559, S E=.051)$, although this

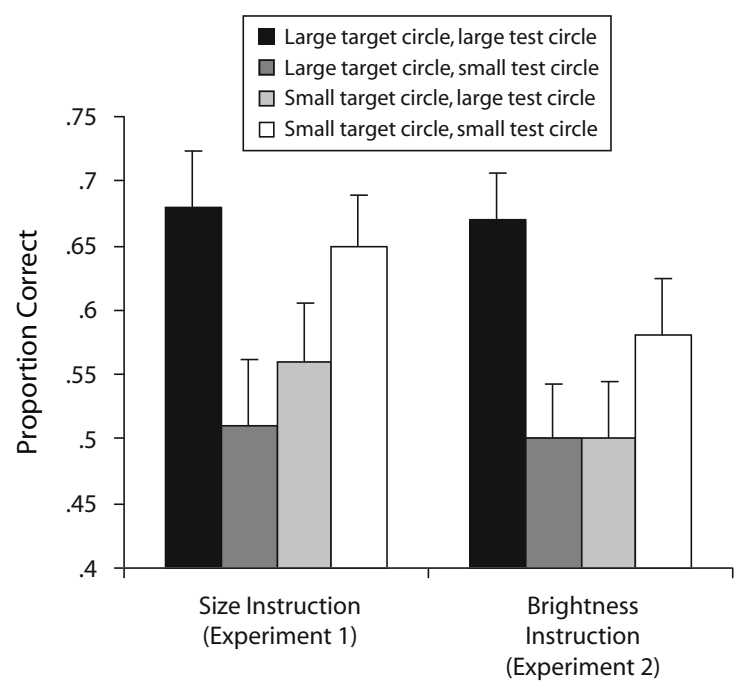

Figure 1. Mean accuracy rates (proportions correct) from Experiments 1 and 2, as a function of size of the attended target circle and size of the correct test circle. Error bars represent standard errors of the means. 
comparison did not reach statistical significance $[t(21)=$ $1.52, p=.144$, two-tailed].

In the present experiment, attention to a single set item led to a modulation in mean estimation accuracy. Importantly, the nature of this modulation was dependent on the size of the attended set item. When attention was directed toward a large circle in the set, participants were subsequently more likely to indicate that the mean size of that set was the same as that of the larger of two test circles (and vice versa following attention to a small circle in the set). This finding suggests that the mean estimations are susceptible to processing differences between set members, either because attention to an item led to an overrepresentation of the item in the computation of the mean of the entire set, or because averages were based on a subset of set items, and that the increased probability for the attended target circle to be included in the selected subset led to a bias in mean estimations according to the size of the attended item. The results do not support the notion that the statistical properties of a set of items can be extracted automatically, since this should not have led to the observed systematic modulation of mean estimations as a function of the size of the attended item.

It was, however, possible that performance on the mean estimation task not only reflected estimations of mean size, but was also affected by the attention manipulation in a more direct fashion, so that observers were more likely to choose the smaller test circle following the instruction to attend to a small set item in the previous display. Such a tendency might reflect either a response bias or a failure to understand the instructions. Although it seemed unlikely that participants misunderstood the instructions, since they were first trained to perform the mean estimation task in isolation and would have had to change their strategy following introduction of the attention manipulation, the fact that targets were explicitly defined on the basis of size might have boosted any carryover effects between the size of the attended target circle and subsequent mean estimation. We therefore repeated the experiment with an attention manipulation that did not explicitly require participants to search for a small or a large target circle.

\section{EXPERIMENT 2}

Experiment 2 was identical to Experiment 1, apart from the way in which the target circle was defined. Whereas in Experiment 1 participants were required to locate either the smallest or the largest circle in the set of nine circles, in Experiment 2 the target circle was presented with a different brightness level from that of the rest of the set, and participants were instructed to report the location of this target. On half the trials, the target was the smallest circle in the set; on the other half of the trials, it was the largest. We argued that by making the size of the target circle an incidental property, rather than the one that defined the target, any strategy effects that might have contributed to the interaction found in Experiment 1 should be attenuated, resulting in a weaker effect of target size on mean estimations, once such a strategy would be less likely. If, on the other hand, the observed modulation of mean estimations reflected a tendency for the size of an attended item to affect the extracted set mean, then the same result should occur even for targets defined in a dimension other than size.

As suggested in the introduction, the finding in Experiment 1 that mean estimations were systematically modulated as a function of the size of the attended item can be explained in terms of two mechanisms involving whole-set averaging. On the one hand, the features of the attended item might have weighed more heavily in the computation of the mean. Alternatively, the fact that target search was done explicitly on the basis of size might have led observers to search set items toward one end of the size distribution, at the expense of items at the other end of the distribution. In Experiment 2, observers had no knowledge of whether the target circle was going to be small or large before onset of the set display, making such a strategy a less likely explanation.

\section{Method}

Participants. Twenty undergraduate students from Goldsmiths, University of London (4 males; age range, 18-33; mean age, 20) participated in the experiment in exchange for course credit. All had normal or corrected-to-normal vision. None had taken part in the previous experiment.

Materials and Procedure. Materials and procedure were the same as for Experiment 1, with the following exceptions. In the experimental trials, instead of the cue word, a fixation cross was presented for $500 \mathrm{msec}$. In the set displays, the target circle was presented in light gray, and the remaining eight circles in darker gray. Participants were asked to report the location of the bright circle (left or right).

\section{Results and Discussion}

A data recording error meant that accuracy data on the localization task was only available for 6 participants, whose mean was .97 . Only trials on which the target circle was correctly located were included in the analysis of mean estimation accuracy. Overall mean estimation accuracy (.56) differed significantly from chance [one-sample $t$ test with test value $=.50 ; t(19)=4.06, p<.01]$. Mean estimation accuracies were entered into a 2 (test circle size: large vs. small) $\times 2$ (target circle size: large vs. small) repeated measures ANOVA, with participants as the random factor (see Figure 1, right graph). There was no main effect of test circle size $(F<1)$, suggesting that mean estimation accuracy was statistically equal for large $(M=.582, S E=.037)$ and small $(M=.537, S E=.042)$ test circles. There was a significant main effect of target circle size $[F(1,19)=$ $\left.10.49, M S_{\mathrm{e}}=.004, p<.01\right]$. Mean estimations were more accurate following a large target circle $(M=.584, S E=$ $.016)$ than following a small target circle $(M=.536, S E=$ .017). Importantly, there was again a significant interaction between test circle size and target circle size $[F(1,19)=$ $\left.34.41, M S_{\mathrm{e}}=.009, p<.001\right]$. Mean estimations for large test circles were more accurate following a large target circle $(M=.669, S E=.037)$ than following a small target circle $(M=.496, S E=.042)[t(19)=6.44, p<.001]$. Conversely, mean estimations for small test circles were more accurate following a small target circle $(M=.577$, $S E=.044)$ than following a large target circle $(M=.498$, $S E=.044)[t(19)=3.13, p<.01]$. 
As in Experiment 1, attention toward an item whose size was at one of the extremes of the distribution led to bias in mean estimations in accordance with the size of the attended item. This time, targets were defined by a dimension other than size, making it unlikely that these results were based entirely on an explicit strategy of attending to (in the set display) and choosing (in the test display) either the smallest or the largest item. ${ }^{1}$ Again, the results do not support the notion of automatic extraction of statistical properties and instead suggest either that the features of the attended item had a systematic effect on computation of the mean size involving most of the set items, or that participants selected a subset of the set items on which to base their mean and compared the sizes of the following test circles with this subset mean to perform the mean estimation task. The fact that Experiment 2, in which size was not the target search criterion, produced the same result as did Experiment 1 strongly suggests that the modulation of mean judgments is not the result of a strategy involving selective scanning of the set items at the same end of the size distribution as the target item. Before onset of the set display, observers had no knowledge of whether the target was going to be small or large, so presumably their search would initially have included small and large items at equal probabilities.

\section{GENERAL DISCUSSION}

When participants were instructed to estimate the mean of a set of circles, one member of which was attended to, estimates were smaller when attention was directed to a small set member, and larger when attention was directed to a large set member. This finding occurred when participants were made to attend to the small or large set member with a direct (Experiment 1) and an indirect (Experiment 2) instruction, as well as when set displays were presented for $200 \mathrm{msec}$ (see note 1). These findings strongly suggest that the statistical properties of a set of items are not always extracted automatically, since this should not have led to the observed systematic modulation of mean estimations as a function of the size of the attended item. Instead, it seems either that whole-set averaging is susceptible to processing differences between set members following attention to some, but not other, set members, or that the extracted mean was not based on the entire set, but on a subset of items of which the attended item was very likely to be part.

A mean based on the entire set should be little affected by the size of the attended item, since the mean of the entire set does of course not differ as a function of which item is attended to. Even if attention has an effect on averaging of the entire set, as suggested previously by showing that set averaging is less accurate when attention is focused locally, rather than distributed globally across the display (Chong \& Treisman, 2005a), then estimation accuracy should show a nonspecific reduction following our attention manipulation, rather than the observed interaction between the size of the target item and the direction of the mean estimation bias.

Instead, these findings support the alternative explanation of set averaging effects in terms of subset averaging.
In the simulations discussed above (Myczek \& Simons, 2008), subsets were selected at random, which meant that, on average, subsets had a mean size similar to that of the entire set. As a result, simulations based on subset averaging of one or two items were very similar to the performance of participants who were instructed to average the entire set, and some previous findings of set averaging (e.g., those of Ariely, 2001) may be explained similarly. In the present study, by directing attention to either the smallest or the largest set item, we ensured that the subset always included an item from one of the two extremes of the distribution. By biasing the sampling in this way, we consistently found evidence that mean estimations were not as expected if they were based on the entire set, but instead seemed to be based on a smaller subset of items.

Alternatively, the present findings may reflect statistical processing of the entire set, and attention to a particular set member may bias the computation of the statistical parameters of a set. On this view, mean estimations are still based on the entire set, but an attended item may make a disproportional contribution to the extracted set parameters. Our data suggest that the most plausible version of the whole-set averaging account is one where the properties of the attended item have more weight in the computation of the mean size of the set than do the properties of the remaining nonattended items. It may even be that attention to a particular set item leads to the formation of two separate representations, one for the attended item and one for the remaining items. If these two representations are given roughly equal weights in the estimation of the mean of the entire set, then the size of the attended item will account for more than its objective weight relative to the number of set items.

The notion that the attention instruction to search for a small or large item could lead to a systematic preference to scan smaller or larger set items, leading to the observed modulation of mean judgments, is unlikely, given that mean judgments were equally affected regardless of whether the search criterion was prespecified as size (Experiment 1) or as brightness (Experiment 2). Given that our results can be accommodated by a model of flexible whole-set averaging and by a model of subset averaging, it remains possible that mean size can be derived through either mechanism, and further work will be needed in order to establish under which conditions observers are more likely to rely on the short-cut strategy of subset averaging. Similarly, the present results are based on the stimulus dimension of size, and it remains to be investigated whether the same applies to other stimulus dimensions for which there is evidence of statistical representation, including orientation and motion speed and direction.

To summarize, we found that mean judgments were systematically affected by the size of the attended set member, so that the set mean was estimated to be larger following attention to a large set member and smaller following attention to a small set member. This effect occurred regardless of whether attention was directed explicitly with the instruction to attend to the smallest or to the largest item (Experiment 1), or implicitly with the instruction to attend to the item that had different brightness from that 
of the rest of the set (Experiment 2). Clearly, extracting the statistical parameter of mean size was sensitive to our attention manipulation, making a model of automatic set averaging unlikely to account for our data. An explanation of these findings in terms of whole-set averaging is possible, although such an explanation calls for a mechanism of flexible weights, so that the value of an attended set member can influence the set mean disproportionately. Our findings can also be explained in terms of subset averaging: Mean estimations are based on a subset of items, which are usually selected randomly, leading to mean estimations that are very similar to the mean of the whole set. However, an attended set member will have an increased probability to be included in the subset, and our systematic manipulation of the size of the attended item will lead to mean estimations that are shifted toward the size of the attended item and away from the whole set mean, just as we found.

\section{AUTHOR NOTE}

The authors thank Lorena Deuker for running the experiment with brief presentations. Also thanks to Dan Simons, Sang Chul Chong, Anne Treisman, and an anonymous reviewer for helpful comments. Correspondence concerning this article should be addressed to J. W. de Fockert, Department of Psychology, Goldsmiths, University of London, New Cross, London SE14 6NW, England (e-mail: j.de-fockert@gold.ac.uk).

\section{REFERENCES}

ARIELY, D. (2001). Seeing sets: Representation by statistical properties. Psychological Science, 12, 157-162.

Atchley, P., \& Andersen, G. (1995). Discrimination of speed distributions: Sensitivity to statistical properties. Vision Research, $\mathbf{3 5}$, 3131-3144.

Chong, S. C., \& Treisman, A. (2003). Representation of statistical properties. Vision Research, 43, 393-404.

Chong, S. C., \& Treisman, A. (2005a). Attentional spread in the statistical processing of visual displays. Perception \& Psychophysics, 67, 1-13.
Chong, S. C., \& Treisman, A. (2005b). Statistical processing: Computing the average size in perceptual groups. Vision Research, 45, 891-900.

DAKIN, S., \& WATT, R. J. (1997). The computation of orientation statistics from visual texture. Vision Research, 37, 3181-3192.

MyczeK, K., \& Simons, D. J. (2008). Better than average: An alternative to statistical summary representations for rapid judgments of average size. Perception \& Psychophysics, 70, 772-778.

Parkes, L., Lund, J., Angelucci, A., Solomon, J., \& Morgan, M. (2001). Compulsory averaging of crowded orientation signals in human vision. Nature Neuroscience, 4, 739-744.

Schneider, W., Eschman, A., \& Zuccolotto, A. (2002). E-Prime reference guide. Pittsburgh, PA: Psychology Software Tools.

WatamaniuK, S. N. J., \& Duchon, A. (1992). The human visual system averages speed information. Vision Research, 32, 931-942.

Williams, D. W., \& SeKuler, R. (1984). Coherent global motion percepts from stochastic local motions. Vision Research, 24, 55-62.

\section{NOTE}

1. In Experiments 1 and 2, set displays were presented for 1,000 msec, making it possible and likely that participants made eye movements toward the target location in the set display. Although fixating the target circle may not have invalidated the findings in Experiments 1 and 2, we investigated whether the same bias would occur when only covert attention, and not eye position, was directed toward a bright target circle, by presenting displays for $200 \mathrm{msec}$ in an experiment with 6 new participants. Mean accuracy on the localization task was .90 . There was again a significant interaction between test circle size and target circle size $\left[F(1,5)=8.78, M S_{\mathrm{e}}=.014, p<.05\right]$. Mean estimations for large test circles were more accurate following a large target circle $(M=.723$, $S E=.050)$ than following a small target circle $(M=.563, S E=.069)$ $[t(5)=2.54, p=.052$, two-tailed]. Conversely, mean estimations for small test circles were marginally more accurate following a small target circle $(M=.742, S E=.066)$ than following a large target circle $(M=$ $.617, S E=.066)[t(5)=2.04, p=.097$, two-tailed $]$. The interaction was significant with only 6 participants, which suggests a particularly robust effect, possibly because the brief presentation duration of the sets either increased the relative salience of the attended item or further necessitated the use of the shortcut strategy of subset averaging.

(Manuscript received November 14, 2006; revision accepted for publication January 17,2008 .) 